\title{
Endogenous Maternal Lipids and Supplementation with Vitamin E Isoform Regulate Neonatal Dendritic Cells during Development of Allergic Disease
}

Kiet Tat ${ }^{1} \&$ Joan Cook-Mills ${ }^{2}$

${ }^{1}$ Indiana University School of Medicine, ${ }^{2}$ Indiana University School of Medicine, Department of Pediatrics

Background and Hypothesis: $C D\|l b+C D\| c+$ dendritic cells (DCs) play a role in the development of allergic disease. It has been shown that of the vitamin $E$ isoforms, $\alpha$-Tocopherol decreases and $y$-Tocopherol increases the generation of bone marrow-derived CD11b+CD11c+ DCs in vivo. And, in vivo experiments have also shown that $\beta$-glucosylceramides, endogenous maternal lipids, increase the neonate proliferation of this same subset of DCs. The mechanism for $\beta$-glucosylceramide regulation of these specific DC subsets is not known. Furthermore, it is also not known how vitamin $E$ isoforms regulate $D C$ development and differentiation. We determined whether $\alpha$-tocopherol decreases and $y$-tocopherol increases responses to $\beta$ glucosylceramide by regulating Protein Kinase C (PKC) activation during CDIlb+CDIlc+ DC differentiation and proliferation.

Project Methods: Cultured bone marrow cells (harvested from mice) were treated with lipid metabolites with and without supplementation of tocopherol isoforms, immunolabeled with antibodies that define DCs and with antibodies that detect active auto phosphorylated forms of PKC. Then, these cells were analyzed using flow cytometry.

Results: In vitro $\beta$-glucosylceramide elevated DC PKC $/ \beta$ activity during $C D I l b+C D \| l c+D C$ differentiation and proliferation/activation. Furthermore, these effects of $\beta$-glucosylceramide on DC PKC $\alpha / \beta$ activity were blocked by $\alpha$-Tocopherol and elevated by $\mathrm{Y}$-Tocopherol.

Potential Impact: These data provides a better understanding of how maternal $\beta$ glucosylceramide and dietary supplementation with vitamin $E$ isoforms regulate DC proliferation and differentiation and ultimately development of allergic inflammation in offspring of allergic mothers. 